Math. Model. Nat. Phenom.

Vol. 2, No. 2, 2007, pp. 126-141

\title{
Atherosclerosis Initiation Modeled as an Inflammatory Process
}

\author{
N. El Khatib ${ }^{1}$, S. Génieys and V. Volpert \\ Université Lyon 1, Institut Camille Jordan, \\ CNRS UMR 5208, 69622 Villeurbanne, France
}

\begin{abstract}
In this work we study the inflammatory process resulting in the development of atherosclerosis. We develop a one- and two-dimensional models based on reaction-diffusion systems to describe the set up of a chronic inflammatory response in the intima of an artery vessel wall. The concentration of the oxidized low density lipoproteins (ox-LDL) in the intima is the critical parameter of the model. Low ox-LDL concentrations do not lead to a chronic inflammatory reaction. Intermediate ox-LDL concentrations correspond to a bistable system and can lead to a travelling wave propagation corresponding to a chronic inflammatory reaction. In this case the disease development depends on the initial condition. If the concentration of monocytes in the intima is sufficiently high, which can be caused by an inflammation related to other factors, then the development of atherosclerosis can start. Otherwise, the system returns to the stable disease free equilibrium. High ox-LDL concentrations correspond to a monostable system and even a small perturbation of the non inflammatory case leads to a travelling wave propagation which corresponds to a chronic inflammatory response.
\end{abstract}

Key words: atherosclerosis, mathematical modelling, travelling waves AMS subject classification: $35 \mathrm{~K} 57,92 \mathrm{C} 45$

\section{Atherogenesis mechanism}

During last decades, several theories about pathogenesis of the atherosclerosis have been developed. None of these theories can explain the whole process because of the big number

\footnotetext{
${ }^{1}$ Corresponding author. Email: nader@math.univ-lyon.fr
} 
of risk factors that contribute to this process [1]. In spite of these multiple theories, the concept of "atherosclerosis as an inflammatory disease" is now well accepted (see [2] and the references therein).

High plasma concentration of low density lipoprotein (LDL) cholesterol is one of the principal risk factors for atherosclerosis. Its mechanism can be sketched as follows $[2,3]$ : the process of atherosclerosis begins when LDLs penetrate into the intima of the arterial wall where they are oxidized. Oxidized LDL (ox-LDL) in the intima is considered by the immune system as a dangerous substance, hence an immune response is launched:

chemoattractants (which mediate the adhesion of the monocytes to the endothelium and the penetration of the monocytes through the endothelium) are released and endothelial cells are activated so that monocytes circulating in the blood adhere to the endothelium and then they penetrate to the arterial intima. Once in the intima, these monocytes are converted into macrophages.

The macrophages phagocytose the ox-LDL but this eventually transform them into foam cells (lipid-ladden cells) which in turn have to be removed by the immune system. In the same time they set up a chronic inflammatory reaction (auto-amplification phenomenon): they secrete pro-inflammatory cytokines (TNF- $\alpha$, IL-1) which increase endothelial cells activation, promote the recruitment of new monocytes and support the production of new pro-inflammatory cytokines.

This auto-amplification phenomenon is compensated by an anti-inflammatory phenomenon mediated by the anti-inflammatory cytokines (IL-10) which inhibit the production of pro-inflammatory cytokines (biochemical anti-inflammation). Next, the inflammation process involves the proliferation and the migration of smooth muscle cells to create a fibrous cap over the lipid deposit which isolates this deposit center from the blood flow (mechanical anti-inflammation).

This mechanical inhibition of the inflammation may become a part of the disease process. Indeed the fibrous cap changes the geometry of the vasculature and modifies the blood flow. The interaction between the flow and the cap may lead to a thrombus, or to the degradation and rupture of the plaque liberating dangerous solid parts in the flow $[4,5]$. In this study we do not address the fluid-structure interaction between the blood flow and the plaque, and only consider the set up of the chronic inflammatory reaction with its biochemical and mechanical inhibitions. We provide a simplified model of the reactions arising in the arterial intima. This model allows us to give the following biological interpretation: at low LDL concentrations the auto-amplification phenomenon does not set up and no chronic inflammatory reaction occurs. At intermediate concentrations a perturbation of the non inflammatory state may lead to the chronic inflammation, but it has to overcome a threshold for that. Otherwise the system returns to the disease free state. At large LDL concentrations, even a small perturbation of the non inflammatory state leads to the chronic inflammatory reaction.

In Section 2 we introduce and study analytically and numerically a one-dimensional model of atherosclerosis development. In Section 3 we consider a two-dimensional model. The latter will allow us to describe in more detail the recruitment of monocytes from the blood 
flow. Numerical simulations show that we recover the results of the $1 D$ model when the domain is thin enough.

\section{One-dimensional model}

We consider here a reaction-diffusion system of equations on an interval representing the arterial intima:

$$
\left\{\begin{aligned}
\frac{\partial M}{\partial t} & =d_{1} \frac{\partial^{2} M}{\partial x^{2}}+f_{1}(A)-\lambda_{1} M \\
\frac{\partial A}{\partial t} & =d_{2} \frac{\partial^{2} A}{\partial x^{2}}+f_{2}(A) M-\lambda_{2} A
\end{aligned}\right.
$$

for $x \in[0, L]$. Here $M$ is the density of immune cells (monocytes, macrophages) and $A$ is the density of the cytokines secreted by the immune cells. The function $f_{1}(A)$ models the recruitment of the immune cells from the blood flow, promoted by the inflammatory cytokines:

$$
f_{1}(A)=\frac{\alpha_{1}+\beta_{1} A}{1+A / \tau_{1}},
$$

where $\alpha_{1}=f_{1}(0)$ corresponds to the beginning of the inflammation, that is the recruitment of monocytes due to the presence of ox-LDL, promoted by endothelial adhesion molecules, chemoattractants and growth factors.

The factor $\beta_{1}$ represents the auto-amplification of the recruitment of monocytes due to the inflammatory cytokines secreted by the monocytes themselves. The factor $1+A / \tau_{1}$ represents the mechanical saturation of the recruitment of $M$ i.e. the effect of the fibrous cap created by smooth muscle cells, with $\tau_{1}$ being the characteristic time for the fibrous cap formation. Although this phenomenon is quite complex and involves proliferation and migration of smooth muscle cells, we only describe it as a saturation factor in this simple model.

The term $f_{2}(A) M$ models the cytokines production rate, with

$$
f_{2}(A)=\frac{\alpha_{2} A}{1+A / \tau_{2}}
$$

where $\alpha_{2} A$ represents the secretion of pro-inflammatory cytokines promoted by the pro-inflammatory cytokines themselves and $1+A / \tau_{2}$ represents the inhibition of the pro-inflammatory cytokines secretion mediated by the anti-inflammatory cytokines, with $\tau_{2}$ being the necessary time for this inhibition to act.

The terms $-\lambda_{1} M$ and $-\lambda_{2} A$ represent the degradation of the immune cells $M$ and the cytokines $A$ respectively, $d_{1} \frac{\partial^{2} M}{\partial x^{2}}$ and $d_{2} \frac{\partial^{2} A}{\partial x^{2}}$ describe their diffusion (or cell displacement) in the intima. 
All the parameters of the model, $\alpha_{1}, \beta_{1}, \tau_{1}, \alpha_{2}, \tau_{2}, \lambda_{1}, \lambda_{2}, d_{1}$ and $d_{2}$ are assumed to be non-negative. Besides, for $f_{1}$ to be an increasing function of $A$, we impose the condition

$$
\tau_{1}>\frac{\alpha_{1}}{\beta_{1}}
$$

\section{$2.1 \quad$ Kinetic system}

In order to determine the conditions for the set up of the inflammatory reaction, we first study only the reaction part of system (2.1), that is we consider the kinetic system of equations

$$
\left\{\begin{aligned}
\frac{d M}{d t} & =f_{1}(A)-\lambda_{1} M, \\
\frac{d A}{d t} & =f_{2}(A) M-\lambda_{2} A .
\end{aligned}\right.
$$

\subsubsection{Equilibrium points}

The isoclines of system (2.1) are defined by the equations

$$
\left\{\begin{array}{r}
f_{1}(A)-\lambda_{1} M=0 \\
f_{2}(A) M-\lambda_{2} A=0
\end{array}\right.
$$

or introducing the notations $F_{1}$ and $F_{2}$,

$$
\left\{\begin{array}{l}
M=F_{1}(A) \equiv \frac{f_{1}(A)}{\lambda_{1}}, \\
M=F_{2}(A) \equiv \frac{\lambda_{2} A}{f_{2}(A)} .
\end{array}\right.
$$

The number of equilibrium points depends on the parameters. We present here different cases and refer to the appendix A for the detailed computations:

The point $E_{0}=\left(0, \frac{\alpha_{1}}{\lambda_{1}}\right)$ is an equilibrium point for any values of the parameters. The existence of other equilibrium points is determined by the equation $F_{1}(A)=F_{2}(A)$ :

$$
\frac{\lambda_{2}}{\alpha_{2} \tau_{1} \tau_{2}} A^{2}+\left(\frac{\lambda_{2}}{\alpha_{2}}\left(\frac{1}{\tau_{1}}+\frac{1}{\tau_{2}}\right)-\frac{\beta_{1}}{\lambda_{1}}\right) A+\frac{\lambda_{2}}{\alpha_{2}}-\frac{\alpha_{1}}{\lambda_{1}}=0
$$

- If $\frac{\lambda_{2}}{\alpha_{2}}<\frac{\alpha_{1}}{\lambda_{1}}$, there is only one positive solution of equation (2.4), denoted by $A_{r}$. We denote by $E_{r}=\left(A_{r}, M_{r}\right)$ the corresponding equilibrium. This case is represented in figure 1.

- Let $\frac{\alpha_{1}}{\lambda_{1}}<\frac{\lambda_{2}}{\alpha_{2}}<\frac{\beta_{1} \tau_{1}}{\lambda_{1}}$. 
- If $0<\frac{\lambda_{2}}{\alpha_{2} \tau_{2}}<\left(\sqrt{\frac{\beta_{1}}{\lambda_{1}}-\frac{\alpha_{1}}{\tau_{1} \lambda_{1}}}-\sqrt{\frac{\lambda_{2}}{\alpha_{2} \tau_{1}}-\frac{\alpha_{1}}{\tau_{1} \lambda_{1}}}\right)^{2}$, then there exist two positive solutions of equation (2.4), denoted by $A_{l}$ and $A_{r}$, where $A_{l}<A_{r}$. The corresponding equilibrium points are denoted by $E_{l}=\left(A_{l}, M_{l}\right)$ and $E_{r}=\left(A_{r}, M_{r}\right)$. This case is represented in figure 2 .

- If $\left(\sqrt{\frac{\beta_{1}}{\lambda_{1}}-\frac{\alpha_{1}}{\tau_{1} \lambda_{1}}}-\sqrt{\frac{\lambda_{2}}{\alpha_{2} \tau_{1}}-\frac{\alpha_{1}}{\tau_{1} \lambda_{1}}}\right)^{2}<\frac{\lambda_{2}}{\alpha_{2} \tau_{2}}$, then there is no positive solution to equation (2.4). This case is represented in figure 3 .

- If $\frac{\beta_{1} \tau_{1}}{\lambda_{1}}<\frac{\lambda_{2}}{\alpha_{2}}$ there is no positive solution to equation (2.4).

\subsubsection{Stability analysis}

- The stability of $E_{0}$ is determined by the Jacobian matrix

$$
J_{E_{0}}=\left(\begin{array}{cc}
-\lambda_{1} & f_{1}^{\prime}(0) \\
0 & \alpha_{2} \frac{\alpha_{1}}{\lambda_{1}}-\lambda_{2}
\end{array}\right)
$$

whose eigenvalues are $\mu_{1}=-\lambda_{1}<0$ and $\mu_{2}=\alpha_{2} \frac{\alpha_{1}}{\lambda_{1}}-\lambda_{2}$. Denoting $E_{1}=\left(0, \frac{\lambda_{2}}{\alpha_{2}}\right)$, we observe that if $E_{0}$ is situated below $E_{1}$ then $E_{0}$ is a stable equilibrium, otherwise it is unstable.

- The stability of $E_{l}$ is determined by

$$
J_{l}=\left(\begin{array}{cc}
-\lambda_{1} & f_{1}^{\prime}\left(A_{l}\right) \\
f_{2}\left(A_{l}\right) & f_{2}^{\prime}\left(A_{l}\right) \frac{\lambda_{2} A_{l}}{f_{2}\left(A_{l}\right)}-\lambda_{2}
\end{array}\right)
$$

whose determinant is $\operatorname{det}\left(J_{l}\right)=\lambda_{1} \lambda_{2}-\lambda_{1} f_{2}^{\prime}\left(A_{l}\right) \frac{\lambda_{2} A_{l}}{f_{2}\left(A_{l}\right)}-f_{2}\left(A_{l}\right) f_{1}^{\prime}\left(A_{l}\right)$. Since $F_{1}$ is concave and $F_{2}$ is a straight line, it holds $F_{1}^{\prime}\left(A_{l}\right)>F_{2}^{\prime}\left(A_{l}\right)$, that is

$$
\frac{f_{1}^{\prime}\left(A_{l}\right)}{\lambda_{1}}>\frac{\lambda_{2} f_{2}\left(A_{l}\right)-\lambda_{2} A_{l} f_{2}^{\prime}\left(A_{l}\right)}{f_{2}^{2}\left(A_{l}\right)}
$$

which implies $\operatorname{det}\left(J_{l}\right)<0$. Hence $E_{l}$ is unstable.

- The stability of $E_{r}$ is determined by

$$
J_{r}=\left(\begin{array}{cc}
-\lambda_{1} & f_{1}^{\prime}\left(A_{r}\right) \\
f_{2}\left(A_{r}\right) & f_{2}^{\prime}\left(A_{r}\right) \frac{\lambda_{2} A_{r}}{f_{2}\left(A_{r}\right)}-\lambda_{2}
\end{array}\right) .
$$


The condition $F_{1}^{\prime}\left(A_{r}\right)<F_{2}^{\prime}\left(A_{r}\right)$ leads to $\operatorname{det}\left(J_{r}\right)>0$.

In order to determine the sign of the eigenvalues, we study the trace of $J_{r}$ :

$$
\operatorname{tr}\left(J_{r}\right)=-\lambda_{1}-\lambda_{2}+f_{2}^{\prime}\left(A_{r}\right) \frac{\lambda_{2} A_{r}}{f_{2}\left(A_{r}\right)} .
$$

The condition $F_{1}^{\prime}\left(A_{r}\right)<F_{2}^{\prime}\left(A_{r}\right)$ leads to

$$
f_{2}^{\prime}\left(A_{r}\right) \frac{\lambda_{2} A_{r}}{f_{2}\left(A_{r}\right)}<\lambda_{2}-\frac{f_{1}^{\prime}\left(A_{r}\right) f_{2}\left(A_{r}\right)}{\lambda_{1}}
$$

which implies $\operatorname{tr}\left(J_{r}\right)<0$. Therefore $E_{r}$ is a stable equilibrium.

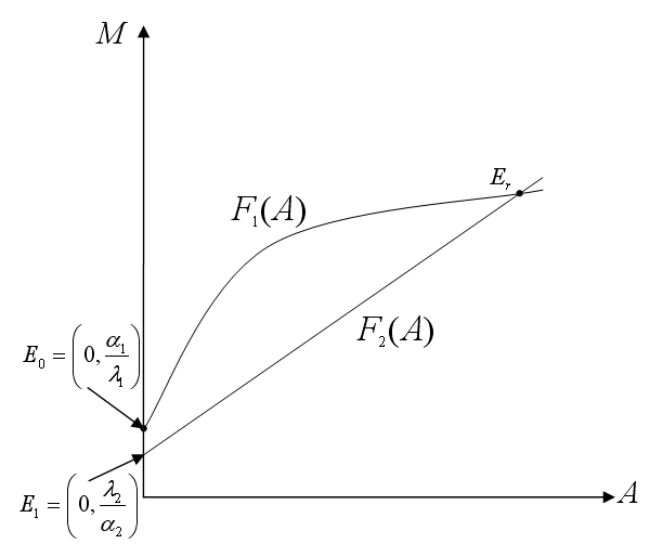

Figure 1: The case $\frac{\lambda_{2}}{\alpha_{2}}<\frac{\alpha_{1}}{\lambda_{1}}$ : two equilibrium points, $E_{0}$ is unstable and $E_{r}$ is stable (the monostable case).

\subsubsection{Biological interpretation}

The equilibrium points of the kinetic system admit the following biological interpretation: $E_{0}$ (no cytokines and low concentration of immune cells) corresponds to the non inflammatory state, whereas $E_{r}$ (large concentrations of cytokines and immune cells) corresponds to the inflammatory state; $E_{0}$ can be stable or unstable, and $E_{r}$ is always stable when it exists.

The intermediate equilibrium $E_{l}$ is always unstable when it exists. It represent a threshold the system has to overcome in order to move from the non inflammatory state $E_{0}$ to the inflammatory one $E_{r}$.

The critical values for the existence and stability of these equilibria were given in terms of the parameters $\frac{\lambda_{2}}{\alpha_{2}}$ and $\frac{\lambda_{2}}{\alpha_{2} \tau_{2}}$ in the last two subsections. The biological interpretation can be more conveniently given in terms of the parameter $\alpha_{1}$ which corresponds to the ox-LDL concentration. But the computation of the critical values between the different cases in terms of $\alpha_{1}$ would be more difficult: 


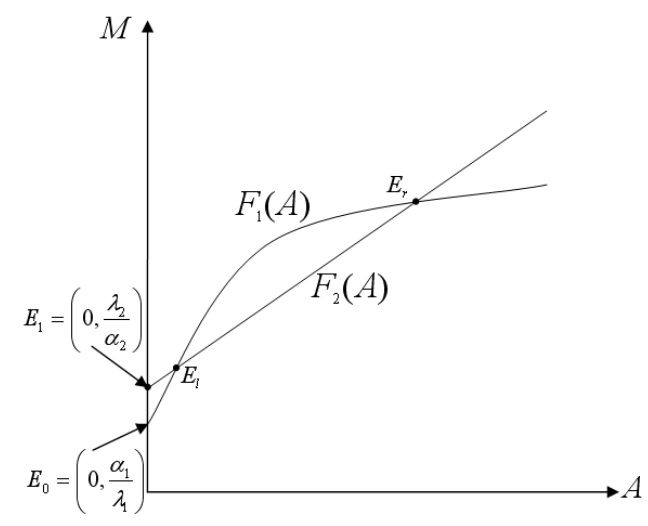

Figure 2: The case $\frac{\alpha_{1}}{\lambda_{1}}<\frac{\lambda_{2}}{\alpha_{2}}<\frac{\beta_{1} \tau_{1}}{\lambda_{1}}$ and $0<\frac{\lambda_{2}}{\alpha_{2} \tau_{2}}<\left(\sqrt{\frac{\beta_{1}}{\lambda_{1}}-\frac{\alpha_{1}}{\tau_{1} \lambda_{1}}}-\sqrt{\frac{\lambda_{2}}{\alpha_{2} \tau_{1}}-\frac{\alpha_{1}}{\tau_{1} \lambda_{1}}}\right)^{2}$ : three equilibrium points, $E_{0}$ and $E_{r}$ are stable and $E_{l}$ is unstable (bistable case).

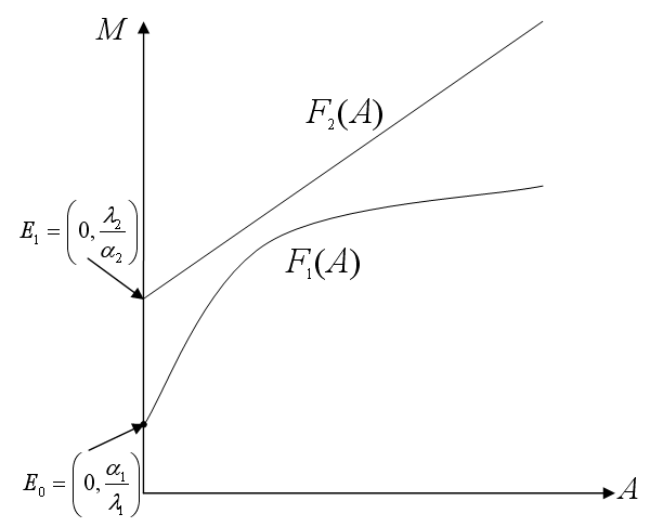

Figure 3: The case $\frac{\alpha_{1}}{\lambda_{1}}<\frac{\lambda_{2}}{\alpha_{2}}<\frac{\beta_{1} \tau_{1}}{\lambda_{1}}$ and $\frac{\lambda_{2}}{\alpha_{2} \tau_{2}}>\left(\sqrt{\frac{\beta_{1}}{\lambda_{1}}-\frac{\alpha_{1}}{\tau_{1} \lambda_{1}}}-\sqrt{\frac{\lambda_{2}}{\alpha_{2} \tau_{1}}-\frac{\alpha_{1}}{\tau_{1} \lambda_{1}}}\right)^{2}$ : only one equilibrium point, $E_{0}$ which is stable. 
- if $\alpha_{1}$ is small (low ox-LDL concentration) $E_{0}$ is the only equilibrium and it is stable. No chronic inflammatory reaction can set up. This case is represented in figure 3.

- if $\alpha_{1}$ is intermediate, there are three equilibrium points: $E_{0}$ and $E_{r}$ are stable and $E_{l}$ is unstable. This case is called bistable case and is represented in figure 2. The system will reach $E_{r}$ if the initial conditions are large enough and $E_{0}$ otherwise. Hence a chronic inflammatory reaction may set up, but for that it has to overcome a threshold.

- if $\alpha_{1}$ is large, there are two equilibrium points: $E_{0}$ is unstable and $E_{r}$ is stable. This case is called monostable case and is represented in figure 1. Even a small perturbation of $E_{0}$ will lead to $E_{r}$. Hence even a small perturbation of the non inflammatory state leads to the set up of a chronic inflammatory reaction.

\subsection{Existence and numerical simulations of travelling waves}

We now investigate the conditions for the propagation of the chronic inflammatory reaction. To this aim we consider the reaction and diffusion phenomena and we turn back to system (2.1). For the theoretical study the space domain will be the entire real line ] $-\infty,+\infty$ [ and for the numerical simulations it will be the segment $[0, L]$. A travelling wave solution of system (2.1) is a particular solution representing a front with constant velocity connecting two equilibria of system (2.3), that is a solution of the form

$$
\left(\begin{array}{c}
A(t, x) \\
M(t, x)
\end{array}\right)=W(x-c t)
$$

where the constant $c$ is a speed of the wave and

$$
\lim _{x \rightarrow \pm \infty} W(x)=W_{ \pm}
$$

with $W_{+}$and $W_{-}$being two equilibria of system (2.3).

Recall that a reaction-diffusion system is called globally monotone if the off-diagonal elements of its Jacobian matrix are positive.

Proposition 2.2.1. System (2.1) is globally monotone.

Proof. Denoting $U(A, M)=\left(\begin{array}{c}U_{1} \\ U_{2}\end{array}\right)(A, M)=\left(\begin{array}{c}f_{1}(A)-\lambda_{1} M \\ f_{2}(A) M-\lambda_{2} A\end{array}\right)$, we have

$$
\frac{\partial U_{1}}{\partial A}(A, M)=f_{1}^{\prime}(A)>0, \quad \frac{\partial U_{2}}{\partial M}(A, M)=f_{2}(A)>0 .
$$

The monotony of the system ensures the existence of travelling wave solutions in the bistable case and in the monostable case (see figures 1 and 2 and Section 2.1.3). The uniqueness of waves holds in the bistable case but not in the monostable one (see e.g. [6], 
Theorem 1.1 and Theorem 1.2, Chapter 3). For system (2.1), this yields the following two results:

Theorem 2.2.2. In the bistable case, there exists a unique travelling wave solution connecting the non inflammatory state $E_{0}$ and the inflammatory state $E_{r}$, i.e. a constant $c$ and a vector valued function $W(x-c t)$, solution of system (2.1) on the real line ] $-\infty,+\infty$ [ and satisfying equation (2.6) with $W_{-}=E_{r}$ and $W_{+}=E_{0}$.

Theorem 2.2.3. In the monostable case, there exists a constant $c^{\star}$ such that $\forall c \in\left[c^{\star},+\infty[\right.$, there exists a travelling wave solution of velocity $c$ connecting the non inflammatory state $E_{0}$ and the inflammatory state $E_{r}$, i.e. a vector valued function $W(x-c t)$, solution of system (2.1) on the real line] $-\infty,+\infty[$ and satisfying equation (2.6) with $W_{-}=E_{r}$ and $W_{+}=E_{0}$.

\subsubsection{Numerical simulations}

We illustrate the previous results by numerical simulations. System (2.1) on the 1D interval $[0, L]$ with the homogeneous Neumann boundary conditions is solved using finite differences. The diffusion term is discretized implicitly and the reaction term explicitly. We first simulate the bistable case. The values of the parameters are $L=1, \alpha_{1}=2, \beta_{1}=8$, $\tau_{1}=1, \lambda_{1}=1, d_{1}=1, \alpha_{2}=7, \tau_{2}=13 / 2, \lambda_{2}=26$ and $d_{2}=1$. The system exhibits a threshold effect: a small perturbation of $E_{0}$ does not lead to the propagation of a chronic inflammation (figure 4) whereas a large perturbation of $E_{0}$ does (figure 5).

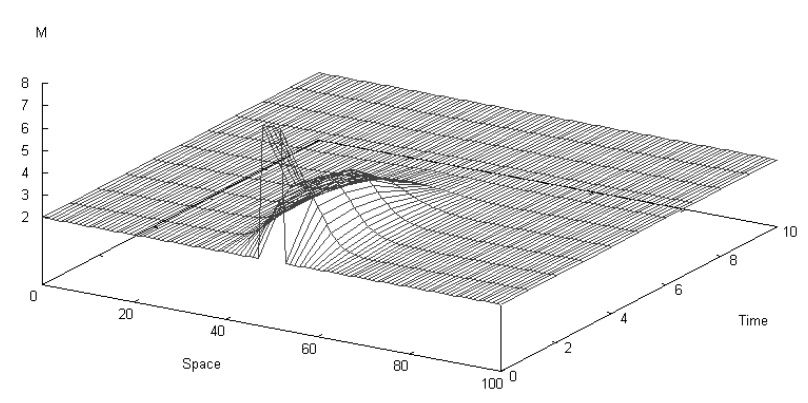

Figure 4: Threshold effect in the bistable case. A small perturbation of the non inflammatory state does not lead to the propagation of a chronic inflammatory reaction.

We then consider the monostable case. The values of the parameters are $L=1, \alpha_{1}=2$, $\beta_{1}=8, \tau_{1}=1, \lambda_{1}=1, d_{1}=1, \alpha_{2}=1, \tau_{2}=42 / 43, \lambda_{2}=1$ and $d_{2}=1$. There is no threshold effect any more. Even small perturbation of $E_{0}$ leads to the propagation of a chronic inflammation.

Since the monotonicity of the system ensures the existence of travelling waves, the conclusions about the onset of a chronic inflammatory reaction is valid also for its propagation: for large ox-LDL concentration, even a small perturbation of the non 


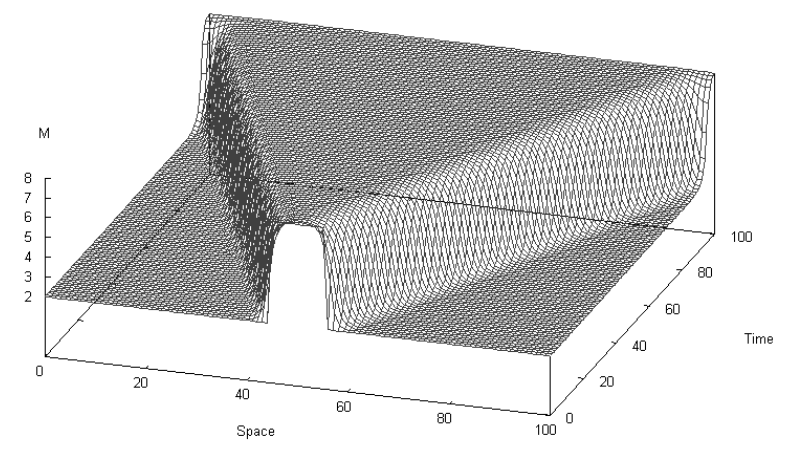

Figure 5: Wave propagation in the bistable case. A large perturbation of the non inflammatory state leads to the propagation of a chronic inflammatory reaction.

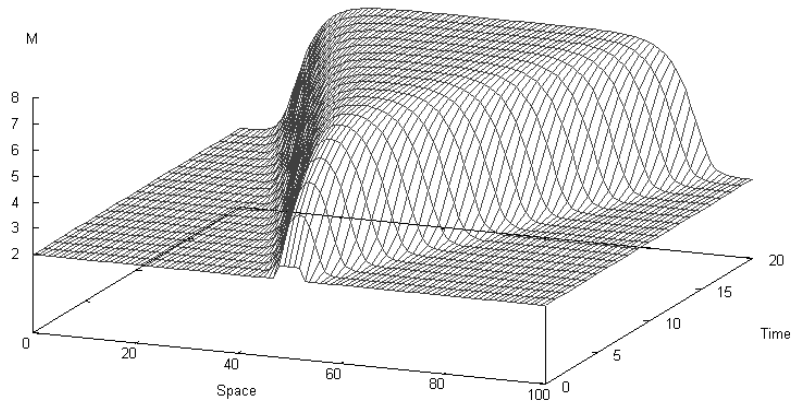

Figure 6: Monostable case. Even a small perturbation of the non inflammatory state leads to the propagation of a chronic inflammatory reaction. 
inflammatory state leads to the development and propagation of the chronic inflammation, whereas for intermediate ox-LDL concentrations it occurs only for large perturbations.

\section{Two-dimensional model}

\subsection{Formulation of the model}

The inflammatory reaction takes place in the arterial intima but it involves the recruitment of monocytes from the blood flow. Hence in a more detailed description, the function $f_{1}(A)$ describing this recruitment should appear as a boundary condition for a model of the intima, and not as a source term.

This is the reason why we developed a two-dimensional model of the intima. The domain is the rectangle $\Omega_{\varepsilon}=[0, L] \times[0, \varepsilon]$. The boundary between the intima and the blood flow is assumed to be the upper horizontal side (figure 7 ). The length of the vertical sides is $\varepsilon$. We expect to recover the qualitative results of the $1 D$-model as $\varepsilon$ tends to 0 (thin domain limit). Indeed the intima is a thin part of the arterial wall accounting for less than $10 \%$ of the total wall thickness. The intima thickness is then much smaller than the typical dimensions of the atherosclerotic lesion. The incoming flux of monocytes decreases proportionally to the width of the domain. Otherwise their concentration in the intima will grow to infinity. This implies the presence of the small parameter in the boundary condition.

We consider the system of equations

$$
\left\{\begin{array}{l}
\frac{\partial M_{\varepsilon}}{\partial t}=d_{1} \Delta M_{\varepsilon}-\lambda_{1} M_{\varepsilon} \\
\frac{\partial A_{\varepsilon}}{\partial t}=d_{2} \Delta A_{\varepsilon}+f_{2}\left(A_{\varepsilon}\right) M_{\varepsilon}-\lambda_{2} A_{\varepsilon}
\end{array}\right.
$$

in $\Omega_{\varepsilon}$. For the immune cells concentration $M_{\varepsilon}$ we consider no flux (homogeneous Neumann) boundary conditions on the vertical and lower horizontal sides, and a non-zero flux depending on $A_{\varepsilon}$ on the upper horizontal side:

$$
\begin{gathered}
\frac{\partial M_{\varepsilon}}{\partial x}(0, y)=\frac{\partial M_{\varepsilon}}{\partial x}(L, y)=0 \text { for } y \in[0, \varepsilon], \frac{\partial M_{\varepsilon}}{\partial y}(x, 0)=0 \text { for } x \in[0, L] \text {, and } \\
\frac{\partial M_{\varepsilon}}{\partial y}(x, \varepsilon)=\frac{f_{1}(A)}{d_{1}} \epsilon \text { for } x \in[0, L] .
\end{gathered}
$$

For the cytokines concentration $A_{\varepsilon}$, we impose homogeneous Neumann boundary conditions everywhere.

The proof of the convergence towards the $1 D$-model in the thin domain limit will be studied in the subsequent work. Formal asymptotic expansion (see appendix B) shows that the prescribed flux for $M_{\varepsilon}$ has to be scaled according to equation (3.8). 


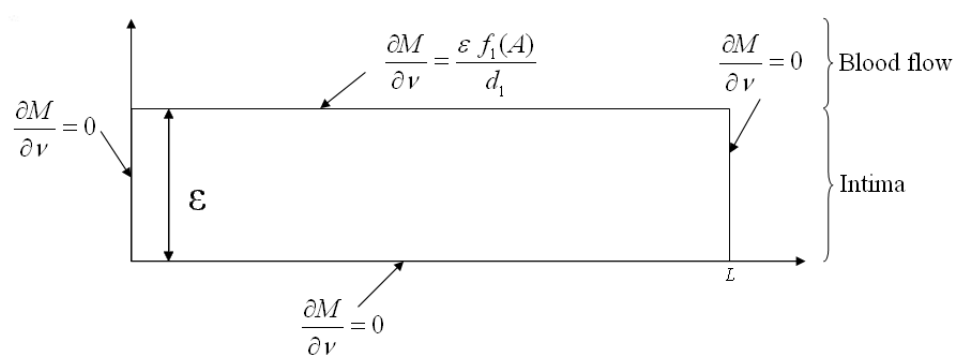

Figure 7: Geometry and boundary conditions of the two-dimensional model.

\subsection{Numerical simulations}

The simulations in the two-dimensional case are performed by using COMSOL Multi-physics (COMSOL (C). With the same values of the coefficients as for the mono-dimensional model we obtain the same qualitative behavior, provided the domain is thin enough.

We first take the parameters from the bistable case of the $1 D$-model: $L=1, \alpha_{1}=2$, $\beta_{1}=8, \tau_{1}=1, \lambda_{1}=1, d_{1}=10^{-3}, \alpha_{2}=7, \tau_{2}=13 / 2, \lambda_{2}=26$ and $d_{2}=10^{-3}$. For $\varepsilon$ small enough $\left(\varepsilon=10^{-3}\right.$ here) the solution $M_{\varepsilon}(x, y)$ weakly depends on $y$ and exhibits the same thresholds effect as for the $1 D$-model. Indeed a small perturbation of the non inflammatory state does not lead to the propagation of the inflammation (not shown), whereas a large enough perturbation does (see figure 3.2).
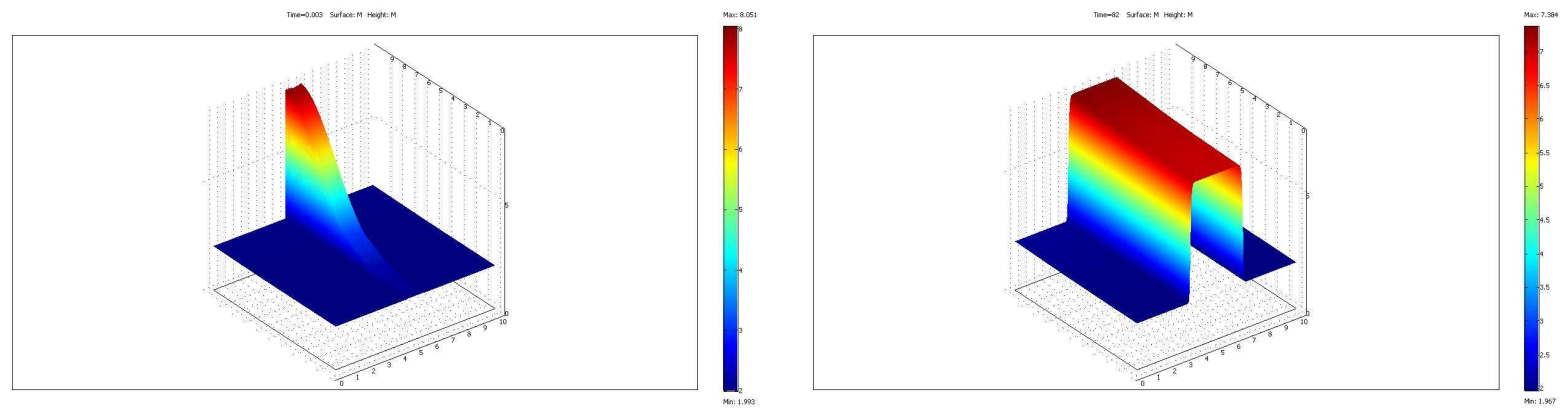

Figure 8: Parameters from the bistable case of the $1 D$-model. The solution weakly depends on $y$ (the $y$-direction is stretched on the figure). A large enough perturbation of the non inflammatory state leads to the propagation of a chronic inflammation. Top: initial condition. Bottom: wave propagation.

We then take the parameters from the monostable case of the $1 D$-model: $L=1, \alpha_{1}=2$, $\beta_{1}=8, \tau_{1}=1, \lambda_{1}=1, d_{1}=10^{-3}, \alpha_{2}=1, \tau_{2}=42 / 43, \lambda_{2}=1, d_{2}=10^{-3}$, and $\varepsilon=10^{-3}$. As for the bistable case $M_{\varepsilon}(x, y)$ weakly depends on the $y$ variable, and we recover the behavior of the $1 D$-model: even a small perturbation of the non inflammatory state leads 
to the propagation of a chronic inflammatory reaction (figure 3.2).
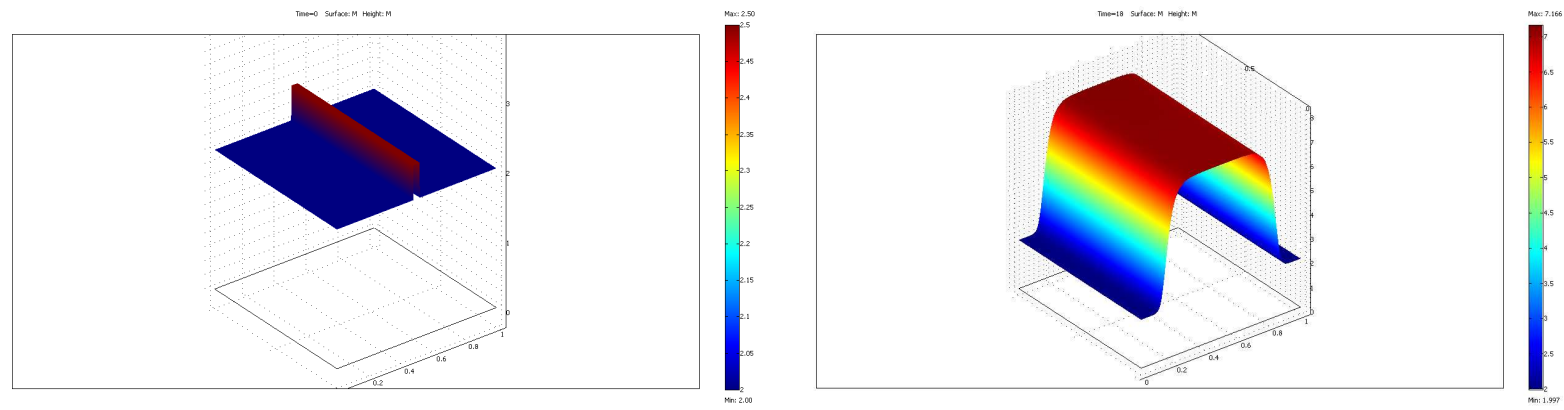

Figure 9: Parameters from the monostable case of the $1 D$-model. The solution weakly depends on $y$ (the $y$-direction is stretched on the figure). Even a small perturbation of the non inflammatory state leads to the propagation of a chronic inflammation. Top: initial condition. Bottom: wave propagation.

\section{Appendix A}

In order to determine the solutions of equation (2.4) it is convenient to rename the cœefficients of $F_{2}: F_{2}(A)=\alpha+\beta A$ where $\alpha=\frac{\lambda_{2}}{\alpha_{2}}$ and $\beta=\frac{\lambda_{2}}{\alpha_{2} \tau_{2}}$. Then equation (2.4) rewrites as

$$
\frac{\beta}{\tau_{1}} A^{2}+\left(\frac{\alpha}{\tau_{1}}+\beta-\frac{\beta_{1}}{\lambda_{1}}\right) A+\alpha-\frac{\alpha_{1}}{\lambda_{1}}=0 .
$$

This is a second order polynomial with respect to $A$ whose discriminant is

$$
\Delta_{A}=\beta^{2}+2\left(2 \frac{\alpha_{1}}{\lambda_{1} \tau_{1}}-\frac{\beta_{1}}{\lambda_{1}}-\frac{\alpha}{\tau_{1}}\right) \beta+\left(\frac{\alpha}{\tau_{1}}-\frac{\beta_{1}}{\lambda_{1}}\right)^{2} .
$$

It is also convenient to introduce $p=\left(\alpha-\frac{\alpha_{1}}{\lambda_{1}}\right) \frac{\tau_{1}}{\beta}$ and $s=\left(\frac{\beta_{1}}{\lambda_{1}}-\frac{\alpha}{\tau_{1}}-\beta\right) \frac{\tau_{1}}{\beta}$, respectively the product and the sum of the roots of equation (4.9).

We discuss the number of solutions to equation (4.9) with respect to the two parameters $\alpha$ and $\beta$.

We distinguish three cases:

- If $\alpha<\frac{\alpha_{1}}{\lambda_{1}}$ then $p<0$ and there is only one positive solution of equation (2.4), denoted by $A_{r}$. 
- Assume

$$
\frac{\alpha_{1}}{\lambda_{1}}<\alpha<\frac{\beta_{1} \tau_{1}}{\lambda_{1}} .
$$

Then $p>0$. We study the sign of $\Delta_{A}$ by considering it as a second order polynomial with respect to the slope $\beta$. Its reduced discriminant is

$$
\Delta_{\beta}^{\prime}=4\left(\frac{\alpha_{1}}{\lambda_{1} \tau_{1}}-\frac{\beta_{1}}{\lambda_{1}}\right)\left(\frac{\alpha_{1}}{\lambda_{1} \tau_{1}}-\frac{\alpha}{\tau_{1}}\right)
$$

and it is positive under assumption 4.10 thanks to equation (2.2). The roots the equation $\Delta_{A}=0$ are

$$
\beta_{-}=\left(\sqrt{\frac{\beta_{1}}{\lambda_{1}}-\frac{\alpha_{1}}{\tau_{1} \lambda_{1}}}-\sqrt{\frac{\alpha}{\tau_{1}}-\frac{\alpha_{1}}{\tau_{1} \lambda_{1}}}\right)^{2}
$$

and

$$
\beta_{+}=\left(\sqrt{\frac{\beta_{1}}{\lambda_{1}}-\frac{\alpha_{1}}{\tau_{1} \lambda_{1}}}+\sqrt{\frac{\alpha}{\tau_{1}}-\frac{\alpha_{1}}{\tau_{1} \lambda_{1}}}\right)^{2} .
$$

Note that under assumption 4.10,

$$
\beta_{-}<\frac{\beta_{1}}{\lambda_{1}}-\frac{\alpha}{\tau_{1}}<\beta_{+}
$$

Hence,

- If $0<\beta<\beta_{-}$, then there exist two solutions of equation (4.9). According to equation (4.11), $s$ is positive, and these two solutions are positive too. We denote them by $A_{l}$ and $A_{r}$, where $A_{l}<A_{r}$.

- If $\beta_{-}<\beta$, then there is no positive solution to equation (4.9) (for $\beta_{-}<\beta<\beta_{+}$, $\Delta_{A}<0$ and equation (4.9) has no real solution, whereas for $\beta_{+}<\beta, \Delta_{A}>0$ but $s<0$ and equation (4.9) has two negative solutions).

- If $\frac{\beta_{1} \tau_{1}}{\lambda_{1}}<\alpha$, then $s$ is negative and there is no positive solution to equation (4.9).

\section{Appendix B}

We formally justify the scaled boundary condition for $M_{\varepsilon}$ in equation (3.8). To this aim, we consider the change of variable $z=y / \varepsilon$, and set $\bar{M}(t, x, z)=M_{\varepsilon}(t, x, y)$. The equation for $\bar{M}$ is then

$$
\frac{\partial \bar{M}}{\partial t}=d_{1} \frac{\partial^{2} \bar{M}}{\partial x^{2}}+\frac{d_{1}}{\varepsilon^{2}} \frac{\partial^{2} \bar{M}}{\partial z^{2}} M-\lambda_{1} \bar{M}
$$


on the stretched domain $\Omega=[0, L] \times[0,1]$, with homogeneous Neumann boundary conditions on the vertical and lower horizontal sides and with

$$
\frac{\partial \bar{M}}{\partial z}(x, 1)=\frac{f_{1}(A)}{d_{1}} \epsilon^{2} \text { for } x \in[0, L]
$$

on the upper horizontal side.

We consider the formal expansion of $\bar{M}$

$$
\bar{M}(t, x, z)=M_{0}(t, x, z)+\varepsilon M_{1}(t, x, z)+\varepsilon^{2} M_{2}(t, x, z)+\ldots
$$

and insert it in equation (5.12) and boundary condition (5.13).

Collecting the coefficients of $\varepsilon^{-2}$ in equation (5.12) yields:

$$
\frac{\partial^{2} M_{0}}{\partial z^{2}}=0
$$

on $\Omega$ with the boundary conditions $\frac{\partial M_{0}}{\partial z}(x, 0)=\frac{\partial M_{0}}{\partial z}(x, 1)=0$. This shows that $M_{0}$ is independent of $z$.

Collecting the coefficients of $\varepsilon^{0}$ in equation (5.12) yields:

$$
\frac{\partial M_{0}}{\partial t}=d_{1} \frac{\partial^{2} M_{0}}{\partial x^{2}}+d_{1} \frac{\partial^{2} M_{2}}{\partial z^{2}}-\lambda_{1} M_{0}
$$

Since $M_{0}$ does not depend on $z$, integrating the latter equation with respect to $z$ between 0 and 1 gives:

$$
\frac{\partial M_{0}}{\partial t}=d_{1} \frac{\partial^{2} M_{0}}{\partial x^{2}}+d_{1} \frac{\partial M_{2}}{\partial z}(x, 1)-\frac{\partial M_{2}}{\partial z}(x, 0)-\lambda_{1} M_{0}
$$

Now collecting the coefficients of $\varepsilon^{2}$ in equation (5.13) gives $\frac{\partial M_{2}}{\partial z}(x, 1)=\frac{f_{1}(A)}{d_{1}}$ and since $\frac{\partial M_{2}}{\partial z}(x, 0)=0$ (homogeneous Neumann conditions on the lower horizontal side), this leads to the $1 D$ equation:

$$
\frac{\partial M_{0}}{\partial t}=d_{1} \frac{\partial^{2} M_{0}}{\partial x^{2}}+f_{1}(A)-\lambda_{1} M_{0}
$$

Hence the solution of the $2 D$-model tends formally to the solution of the $1 D$-model when $\varepsilon$ tends to 0 .

\section{Acknowledgements}

The authors are grateful to John McGregor for discussions on the inflammatory aspect of atherosclerosis, and to Ionel Ciuperca for discussions about thin domains. 


\section{References}

[1] J. Fan, T. Watanabe. Inflammatory Reactions in the Pathogenesis of Atherosclerosis. J. Atheroscler. Thromb., 2003, Vol. 10, 63-71.

[2] R. Ross. Atherosclerosis - An inflammatory disease. New Engl. J. Med., 1999, Vol. 340, 115-26.

[3] B. Osterud, E. Bjorklid. Role of Monocytes in Atherogenesis. Physiol. Rev., 2003, Vol. 83, 1070-1086.

[4] Z.Y. Li, S. Howarth, T. Tang, J.H. Gillard. How Critical Is Fibrous Cap Thickness to Carotid Plaque Stability? A Flow Plaque Interaction Model. Stroke, 2006, Vol. 37, 1195-1196.

[5] Z.Y. Li, S. Howarth, R.A. Trivedi, J.M. U-King-Im, M.J. Graves, A. Brown, L. Wang, J.H. Gillard. Stress analysis of carotid plaque rupture based on in vivo high resolution MRI. J. Biomech., 2006, Vol. 39, No. 14, 2611-2612.

[6] A.I. Volpert, V.A. Volpert, V.A. Volpert. Traveling Wave Solutions of Parabolic Systems. American Mathematical Society, Providence Rhode Island, 2000. 